\title{
Adaptive Command Filtered Backstepping Trajectory Tracking Control of Hexarotor UAV
}

\author{
Chengshun Yang ${ }^{1, *}$ and Zhong Yang ${ }^{2}$ \\ ${ }^{1}$ School of Electric Power Engineering, Nanjing Institute of Technology, Nanjing, China \\ ${ }^{2}$ College of Automation Engineering, Nanjing University of Aeronautics and Astronautics, Nanjing, China
}

\begin{abstract}
In this paper, a hexarotor unmanned aerial vehicle (UAV) is concerned to solve such problems as smaller payload capacity, lack of both hardware redundancy and anti-crosswind capability for quad-rotor. Considering the under-actuated and strong coupling nonlinear system with external disturbance and parameter uncertainty properties of the hexarotor $\mathrm{UAV}$, a nested double-loops trajectory tracking control strategy is proposed. A position error PID controller is designed as the outer-loop controller, of which the task is to compare the desired trajectory with real position of the hexarotor UAV and export the desired attitude angles to the inner-loop. And, an adaptive commandfiltered backstepping controller is designed as the inner-loop controller which makes use of parameter update laws to estimate the disturbances of the hexarotor UAV. The simulation results show that the proposed control strategy enhances the trajectory tracking performance by controlling the external disturbance and parameter uncertainty.
\end{abstract}

Keywords- Hexarotor UAV, trajectory tracking control, backstepping, adaptive, command filter

\section{INTRODUCTION}

Rotary wing type UAVs are classified into multi-rotor type (such as quad-rotor and hexarotor), co-axial helicopter, and traditional helicopter, etc. The design of the vehicle is simpler than for normal helicopters in that the quad-rotor does not use mechanical linkages to vary the rotor blade pitch angle as they spin and this reduces maintenance time and cost. Further, its relatively low-cost feature make it attractive candidates for swarm operations, a field of ongoing research in the UAV community[1-4].

The purpose of this paper is to design a trajectory tracking control system forcing the hexarotor UAV (The structure as shown in Fig.1) to track the desired trajectory accurately. To realize this purpose, the highly stable and nonlinear controllers are required. In consideration of the under-actuated and strong coupling characteristics of the hexarotor UAV, the nested double-loops trajectory tracking control strategy is introduced in this paper. Accordingly, the outer-loop refers to the position loop while the inner-loop refers to the attitude control loop. In the outer-loop, a position error proportional-integral-derivative (PID) controller is developed, of which the task is to compare the desired trajectory with real position of the hexarotor UAV and construct the command signals to the inner-loop.

In the approach of attitude controller design, dynamic inversion[5], feed linearization and sliding mode control[6], model reference adaptive[7] have been widely used.
Backstepping control design[2-4], due to its simple, has become an effective approach for controller design. The rotational dynamics of the hexarotor UAV satisfies the strict feedback form, so it can be used backstepping to design the attitude controller. However, the traditional backstepping control also has several of inadequacies which limit the backstepping technique in practical applications. First, the analytic derivative expressions of virtual control variables are usually overly complicated or unknown especially for systems with uncertain or noise. Second, considering the practical application, the states of the UAV especially for the attitude angles and angular rate are usually needed to limit. For instance, in the inspection of the transmission lines using the hexarotor UAV, in order to facilitate the camera carried by the UAV focusing on the components of the transmission lines, the attitude angles and angular rate of the hexarotor UAV cannot change too much. Third, the problem of the control saturation is not considered $[8,9]$. Especially the last defect, it may lead to serious problems in the actual control systems. If the generated control command is not fully implemented by actuators, the accumulation of errors may lead to the system unstable. However, these problems are not considered in the reference [2-4]. To solve these problems, Farrell, etc. $[10,11]$ introduced a constrained command filter into backstepping control systems. The command filter is used to eliminate the impact of derivative of the virtual control signals and control saturation.

Motivated by the above analysis, an adaptive commandfiltered backstepping attitude controller for the hexarotor UAV is designed to overcome the problems of input and state constraints, avoid calculating the virtual control signal derivative analytically and increase the robustness of the disturbances. The adaptive backstepping method proposed in this paper is a recursive, Lyapunov-based, nonlinear controller design approach which makes use of parameter update laws to estimate the disturbances of the rotational dynamics. With the command filter, it is possible to control the limit of the attitude and angular rate, at the same time under the actuator constraints[10]. And, the derivatives of the pseudo control signal are numerically calculated by the command filter instead of calculating it analytically. At the same time, an auxiliary filter is also introduced to compensate for the command filter error simultaneously satisfying the overall stability requirement.

\section{MATHEMATICAL ModELING}

To simplify the modeling of the hexarotor UAV and make the controller design easier, several reasonable assumptions are 
made: 1) hexarotor UAV is a rigid. Then the nonlinear dynamics can be derived by using Newton-Euler formulas. 2) The structure of the hexarotor UAV is symmetrical with respect to the axes $O x, O y$ and $o z .3$ ) The height between the rotors and the plane of the hexarotor UAV is ignored.



FIGURE I. THE STRUCTURE OF HEXAROTOR UAV AND THE ASSOCIATED FRAMES

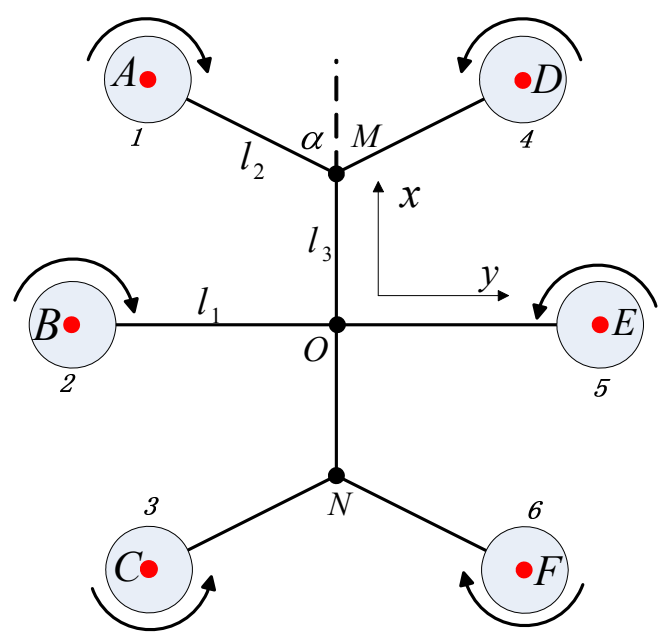

FIGURE II. THE SIMPLE STRUCTURE OF HEXAROTOR UAV

Firstly, two frames have to be defined: a body-fixed frame (B-frame) and an earth-fixed frame (E-frame). Let $\mathbf{B}=\{o x y z\}$ denote the body-fixed frame whose origin $O$ is at the center of mass of the hexarotor and $\mathbf{E}=\left\{o_{g} x_{g} y_{g} z_{g}\right\}$ denote the earthfixed frame, as shown in Fig. 1. Therefore, under the assumption 3), the structure of the hexarotor UAV can be simplified as shown in Fig. 2.

In Fig. $2, l_{1}$ denotes the length of $\mathrm{OB}$ and $\mathrm{OE}, l_{2}$ denotes the length of AM, DM, CN and FN, $l_{3}$ denotes the length of $\mathrm{OM}$ and $\mathrm{ON}, a$ represents the included angle between $\mathrm{AM}$ and OM.

Then, the translational dynamics and the rotational dynamics [12] of the hexarotor UAV can be expressed as

$$
\left\{\begin{array}{l}
\dot{p}=v \\
\dot{v}=-g z_{e}+\frac{T}{m} R z_{e}
\end{array}\right.
$$

$$
\left\{\begin{array}{l}
\dot{\xi}=W \Omega+\beta_{1} D_{1} \\
\dot{\Omega}=-J^{-1}(\Omega \times J \Omega)-J^{-1} G_{a}+J^{-1} \tau^{b}+\beta_{2} D_{2}
\end{array}\right.
$$

In (1), $\boldsymbol{p}=(x, y . z)^{\mathrm{T}}$ and $\boldsymbol{v}=\left(v_{x}, v_{y}, v_{z}\right)^{\mathrm{T}}$ are the position and velocity of the hexarotor UAV in the E-frame, respectively. $g$ is the gravitational acceleration, $T$ is the resulting force in the B-frame (excluding the gravity force) acting on the airframe and $\boldsymbol{z}_{e}=(0,0,1)^{\mathrm{T}}$ is a unit vector expressed in the Eframe. $R \hat{\mathrm{I}} S O(3)$ is the orthogonal rotation matrix to orient the hexarotor UAV and defined as follows

$$
R=\left[\begin{array}{ccc}
c \theta c \psi & s \theta c \psi s \phi-s \psi c \phi & s \theta c \psi c \phi+s \psi s \phi \\
c \theta s \psi & s \theta s \psi s \phi+c \psi c \phi & s \theta s \psi c \phi-c \psi s \phi \\
-s \theta & c \theta s \phi & c \theta c \phi
\end{array}\right]
$$

where $\boldsymbol{\xi}=(f, q, y)^{\mathrm{T}}$ denotes the vector of three Euler angles and $s$. and $c$. are abbreviations for $\sin (\cdot)$ and $\cos (\cdot)$.

And, in (2) $\Omega=(p, q, r)^{\mathrm{T}}$ is the angular rate, $W$ is an Euler matrix [4] and given by

$$
W=\left[\begin{array}{ccc}
1 & \sin \phi \tan \theta & \cos \phi \tan \theta \\
0 & \cos \phi & -\sin \phi \\
0 & \sin \phi \sec \theta & \cos \phi \sec \theta
\end{array}\right]
$$

$J \hat{\mathrm{I}} \mathrm{R}^{3^{\prime}} 3$ is the total inertial matrix of the hexarotor UAV. Under the assumption 2), it is a diagonal positive definite constant matrix expressed in the B-frame. $D_{i},(i=1,2)$ are composite disturbances, including aerodynamic moments, external disturbances and parameter uncertainties. $b_{i},(i=1,2)$ are the input disturbance matrices. And the vector $G_{a}$ expresses the gyroscopic torque given by:

$$
G_{a}=J_{r}\left(\Omega \times z_{e}\right)\left(\sum_{i=3,4,5} \omega_{i}-\sum_{i=1,2,6} \omega_{i}\right)
$$

where $J_{r}$ and $\omega_{i}$ are, respectively, the rotor inertia and the rotor speed.

From (1) and (2), It is obvious that the hexarotor UAV is an under-actuated mechanical system with six degree of freedom (DOF) and four main control inputs. It is characterized by one main control force $T$ generated by the six propellers in the free 
air and three main control torques $\boldsymbol{\tau}^{b}=\left(\tau_{\phi}, \tau_{\theta}, \tau_{\psi}\right)^{\mathrm{T}}$. So, the control force $T$ can be expressed as

$$
T=\sum_{i=1}^{6} f_{i}=b \sum_{i=1}^{6} \omega_{i}^{2}
$$

At the same time, the reactive torque caused by air drag is also generated by each propeller and given by $Q_{i}=d \omega_{i}^{2}$. Thus, the total reactive torque by the six propellers is given as follow:

$$
Q=\sum_{i=3,4,5} Q_{i}-\sum_{i=1,2,6} Q_{i}=d\left(\sum_{i=3,4,5} \omega_{i}^{2}-\sum_{i=1,2,6} \omega_{i}^{2}\right)
$$

where $\omega_{i}$ is the rotor speed, $b$ and $d$ are thrust and drag factors[3], respectively.

Therefore, according to Fig. 2 and (5), it is easy to obtain the airframe torques generated by the six propellers given by:

$$
\tau^{b}=\left[\begin{array}{c}
\tau_{\phi} \\
\tau_{\theta} \\
\tau_{\psi}
\end{array}\right]=\left[\begin{array}{c}
b l_{2} \sin \alpha\left(\omega_{4}^{2}+\omega_{6}^{2}-\omega_{1}^{2}-\omega_{3}^{2}\right)+b l_{1}\left(\omega_{5}^{2}-\omega_{2}^{2}\right) \\
b\left(l_{2} \cos \alpha+l_{3}\right)\left(\omega_{1}^{2}+\omega_{4}^{2}-\omega_{3}^{2}-\omega_{6}^{2}\right) \\
d\left(\omega_{3}^{2}+\omega_{4}^{2}+\omega_{5}^{2}-\omega_{1}^{2}-\omega_{2}^{2}-\omega_{6}^{2}\right)
\end{array}\right]
$$

In order to facilitate the computation of the real control inputs, that is, $\omega_{i},(i=1,2, \cdots, 6),(4)$ and $\tau^{b}$ are put together:

$$
\left(T, \tau_{\phi}, \tau_{\theta}, \tau_{\psi}\right)^{\mathrm{T}} \triangleq M \bar{\omega}
$$

where

$$
\begin{gathered}
M=\left[\begin{array}{cccccc}
b & b & b & b & b & b \\
-b l_{2} \sin \alpha & -b l_{1} & -b l_{2} \sin \alpha & b l_{2} \sin \alpha & b l_{1} & b l_{2} \sin \alpha \\
b\left(l_{2} \cos \alpha+l_{3}\right) & 0 & -b\left(l_{2} \cos \alpha+l_{3}\right) & b\left(l_{2} \cos \alpha+l_{3}\right) & 0 & b\left(l_{2} \cos \alpha+l_{3}\right) \\
-d & -d & d & d & d & -d
\end{array}\right] \\
\bar{\omega}=\left(\begin{array}{llllll}
\omega_{1}^{2} & \omega_{2}^{2} & \omega_{3}^{2} & \omega_{4}^{2} & \omega_{5}^{2} & \omega_{6}^{2}
\end{array}\right)^{\mathrm{T}}
\end{gathered}
$$

\section{TRAJECTORY TRACKING CONTROL STRATEGY}

Considering the under-actuated and strong coupling characteristics of the hexarotor UAV, the nested double-loops trajectory tracking control strategy is introduced. And, the relationship between the outer-loop and inner-loop is established based on the position error PID controller. Then an adaptive command-filtered backstepping controller for the hexarotor UAV is designed to track the desired attitude angles generated by the outer-loop. The controller makes use of parameter update laws to estimate the composite disturbances of the rotational dynamics of the hexarotor UAV. And, an auxiliary filter is also introduced to compensate for the command filter error simultaneously satisfying the overall stability requirement.

To give a clear idea of the overall design procedure, a flow chart is depicted as Fig.3

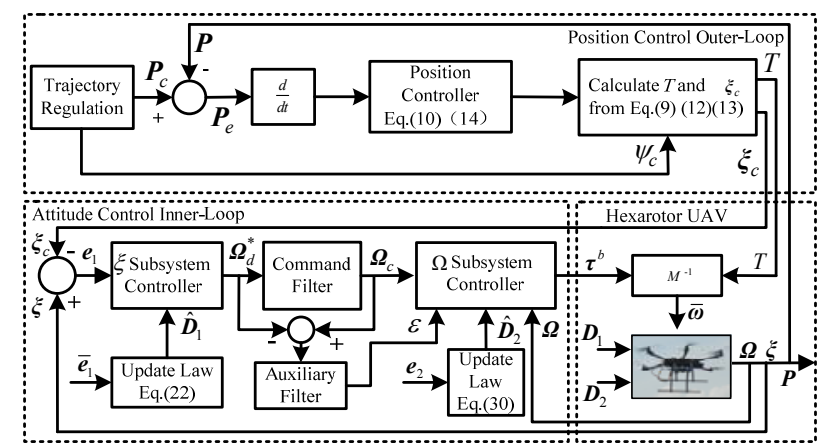

FIGURE III. THE FLOW CHART OF THE DESIGN PROCEDURE

\section{A. Position controller design}

In this section, a position controller based on PID in the outer-loop will be developed, of which the task is to compare the desired trajectory with real position of the hexarotor UAV and construct the desired attitude angles to the inner loop. The translational dynamics (1) will be treated as the plant to the controller which calculates the desired attitude angles and control force based on the desired trajectory.

Based on (1), we have

$$
\ddot{z}=-g+\cos \phi \cos \theta \frac{T}{m}
$$

$$
\begin{aligned}
& \ddot{x}=(\cos \phi \sin \theta \cos \psi+\sin \phi \sin \psi) \frac{T}{m} \\
& \ddot{y}=(\cos \phi \sin \theta \sin \psi-\sin \phi \cos \psi) \frac{T}{m}
\end{aligned}
$$

The altitude subsystem (6) containing vertical force input $T$ which can be linearized by selecting $T$ as

$$
T=\frac{m g}{\cos \phi \cos \theta}+\frac{u}{\cos \phi \cos \theta}
$$

The necessary condition for (9) is $\cos \phi \cos \theta \neq 0$, where $u$, a PD controller, is given by

$$
u=-K_{d} \dot{z}-K_{p}\left(z-z_{d}\right)
$$


where $K_{p}$ and $K_{d}$ are the proportional and the derivative positive gain and $z_{d}$ the desired altitude.

Position subsystem is given by (7) and (8). Let $\dot{x}_{d}$ and $\dot{y}_{d}$ be the desired speed in $\mathrm{x}$ and $\mathrm{y}$ direction, respectively. Then, the errors at desired and actual speed are separately given by

$$
\begin{aligned}
& e_{x}=\dot{x}_{d}-\dot{x} \\
& e_{y}=\dot{y}_{d}-\dot{y}
\end{aligned}
$$

The desired roll and pitch angles in terms of errors between actual and desired speed are, thus, separately given by

$$
\begin{aligned}
& \phi_{c}=\arcsin \left(u_{e_{x}} \sin \psi-u_{e_{y}} \cos \psi\right) \\
& \theta_{c}=\arcsin \left(\frac{u_{e_{x}}}{\cos \phi \cos \psi}-\frac{\sin \phi \sin \psi}{\cos \phi \cos \psi}\right)
\end{aligned}
$$

where, $u_{e_{x}}$ and $u_{e_{y}}$ are

$$
u_{e_{x}}=\frac{K_{x} e_{x} m}{T},-u_{e_{y}}=\frac{K_{y} e_{y} m}{T}
$$

where $K_{x}$ and $K_{y}$ are the positive constants and $T$ is the desired vertical force input by the altitude control.

\section{B. Attitude controller design}

First, a reasonable assumption and a definition are given as follows.

Assumption 1: The derivatives of the disturbances $\dot{D}_{i} \approx 0, i=1,2$. (This means that the disturbances change slowly.)

Definition 1: $\tilde{D}_{i}=D_{i}-\hat{D}_{i}, i=1,2$ are the estimation errors of the disturbances.

Consider the rotational dynamics given by (2), and define, respectively, the attitude and angular rate tracking errors as follows:

$$
\begin{gathered}
e_{1}=\xi-\xi_{c} \\
e_{2}=\Omega-\Omega_{c}
\end{gathered}
$$

where $\boldsymbol{\xi}_{\mathrm{c}}=\left(\phi_{c}, \theta_{c}, \psi_{c}\right)^{\mathrm{T}}$ are the desired attitude angles computed by (12)and (13). $\Omega_{c}$ is the filtered-command of $\Omega_{d}^{*}$ and it will be defined later.
The derivatives of $e_{1}$ and $e_{2}$ are given by

$$
\dot{e}_{1}=W \Omega+\beta_{1} D_{1}-\dot{\xi}_{\mathrm{c}}
$$

$$
\dot{e}_{2}=-J^{-1}(\Omega \times J \Omega)-J^{-1} G_{a}+J^{-1} \tau^{b}+\beta_{2} D_{2}-\dot{\Omega}_{c}
$$

To give a clear idea of the controller design procedure, the following steps are given.

Step 1: In this step, the task is to stabilize (17) with respect to the Layapunov function

$$
\mathrm{V}_{1}=\frac{1}{2} e_{1}^{\mathrm{T}} e_{1}+\frac{1}{2} \tilde{D}_{1}^{\mathrm{T}} \Phi_{1} \tilde{D}_{1}
$$

where $\tilde{D}_{1}=D_{1}-\hat{D}_{1}, \Phi_{1}$ is a positive definite design parameter matrix.

The time derivative of $V_{1}$ with respect to time is given by

$$
\begin{aligned}
\dot{\mathrm{V}}_{1} & =e_{1}^{\mathrm{T}} \dot{e}_{1}+\tilde{D}_{1}^{\mathrm{T}} \Phi_{1} \dot{\tilde{D}}_{1} \\
& =e_{1}^{\mathrm{T}}\left(W \Omega+\beta_{1} D_{1}-\dot{\xi}_{c}\right)-\tilde{D}_{1}^{\mathrm{T}} \Phi_{1} \dot{\hat{D}}_{1}
\end{aligned}
$$

Then, the virtual controller and parameter update law for $D_{1}$ can be designed as

$$
\begin{gathered}
\Omega_{d}^{*}=-W^{-1}\left(c_{1} e_{1}-\dot{\xi}_{c}+\beta_{1} \hat{D}_{1}\right) \\
\dot{\hat{D}}_{1}=\Phi_{1}^{-1} \beta_{1}^{\mathrm{T}} e_{1}
\end{gathered}
$$

where $\Omega_{d}^{*}$ is the desired angular rate and $c_{1}$ is a positive definite matrix to be designed.

Substituting (21) and (22) into (20) yields

$$
\begin{aligned}
\dot{\mathrm{V}}_{1} & =e_{1}^{\mathrm{T}}\left(-c_{1} e_{1}+\beta_{1} \tilde{D}_{1}\right)-\tilde{D}_{1}^{\mathrm{T}} \beta_{1}^{\mathrm{T}} e_{1} \\
& =-e_{1}^{\mathrm{T}} c_{1} e_{1}<0
\end{aligned}
$$

And we can get the conclusion that (21) and (22) can guarantee the stability of (17).

In order to overcome the problems of input and state constraints and avoid calculating the virtual control signal derivative analytically, a constrained command filter is introduced into the procedure of the adaptive backstepping control design. $\Omega_{d}^{*}$ and $\Omega_{c}$ are the input and output of the command filter as described in Fig.4. In addition, the derivate of $\Omega_{c}$, namely $\dot{\Omega}_{c}$ can also be generated by the command filter. 


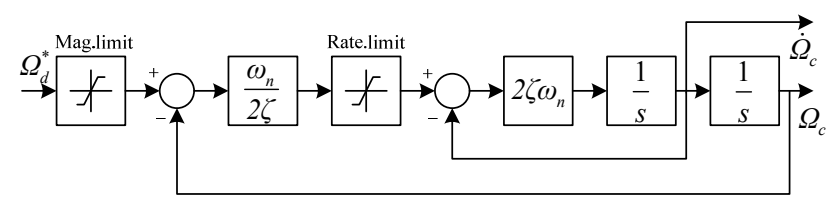

FIGURE IV. COMMAND FILTER BLOCK DIAGRAM

where $\xi$ and $\omega_{n}$ are damping ratio and natural frequency of the command filter, respectively.

Thus, the state space of the command filter can be represented as[11]

$$
\left(\begin{array}{c}
\dot{\Omega}_{c} \\
\ddot{\Omega}_{c}
\end{array}\right)=\left(\begin{array}{c}
\dot{\Omega}_{c} \\
2 \xi \omega_{n}\left(\mathrm{~S}_{R}\left(\frac{\omega_{n}^{2}}{2 \xi \omega_{n}}\left(\mathrm{~S}_{M}\left(\Omega_{d}^{*}\right)-\Omega_{c}\right)\right)-\dot{\Omega}_{c}\right)
\end{array}\right)
$$

where $\mathrm{S}_{M}(\cdot)$ and $\mathrm{S}_{R}(\cdot)$ denote magnitude and rate limited function, and $\mathrm{S}_{M}(\cdot)$ is defined as

$$
\mathrm{S}_{M}(x)= \begin{cases}M, & x \geq M \\ x, & x<|M| \\ -M, & x \leq-M\end{cases}
$$

And, the definition of $\mathrm{S}_{R}(\cdot)$ is similar to $\mathrm{S}_{M}(\cdot)$.

In the interval of linear change of the $\mathrm{S}_{R}(\cdot)$ and $\mathrm{S}_{M}(\cdot)$, the state space of the command filter can be described as

$$
\left(\begin{array}{l}
\dot{\Omega}_{c} \\
\ddot{\Omega}_{c}
\end{array}\right)=\left(\begin{array}{cc}
0 & 1 \\
-\omega_{n}^{2} & -2 \xi \omega_{n}
\end{array}\right)\left(\begin{array}{c}
\Omega_{c} \\
\dot{\Omega}_{c}
\end{array}\right)+\left(\begin{array}{c}
0 \\
\omega_{n}^{2}
\end{array}\right) \Omega_{d}^{*}
$$

It is obviously that the system (23) is a linear stable system. Accordingly, if $\Omega_{d}^{*}$ is bounded, $\Omega_{c}$ and $\dot{\Omega}_{c}$ are bounded and continuous.

At the same time, in consideration of the influence caused by the command filter, an auxiliary filter is also introduced to compensate for the command filter error simultaneously satisfying the overall stability requirement.

$$
\dot{\varepsilon}=-c_{1} \varepsilon+W\left(\Omega_{c}-\Omega_{d}^{*}\right)
$$

Thus, the attitude tracking error and the parameter update law for $D_{1}$ are redefined as follows

$$
\bar{e}_{1}=\xi-\xi_{\mathrm{c}}-\varepsilon
$$

$$
\dot{\hat{D}}_{1}=\Phi_{1}^{-1} \beta_{1}^{\mathrm{T}} \bar{e}_{1}
$$

Step 2: In this step, the task is to design the control torques and achieve $\boldsymbol{\Omega}$ tracking $\boldsymbol{\Omega}_{c}$ asymptotically.

Select the Lyapunov function as

$$
\mathrm{V}_{2}=\frac{1}{2} \bar{e}_{1}^{\mathrm{T}} \bar{e}_{1}+\frac{1}{2} \tilde{D}_{1}^{\mathrm{T}} \Phi_{1} \tilde{D}_{1}+\frac{1}{2} e_{2}^{\mathrm{T}} e_{2}+\frac{1}{2} \tilde{D}_{2}^{\mathrm{T}} \Phi_{2} \tilde{D}_{2}
$$

and the time derivation of $V_{2}$ is given by

$$
\dot{\mathrm{V}}_{2}=\bar{e}_{1}^{\mathrm{T}} \dot{\bar{e}}_{1}+\tilde{D}_{1}^{\mathrm{T}} \Phi_{1} \dot{\tilde{D}}_{1}+e_{2}^{\mathrm{T}} \dot{e}_{2}+\tilde{D}_{2}^{\mathrm{T}} \Phi_{2} \dot{\tilde{D}}_{2}
$$

where $\tilde{D}_{2}=D_{2}-\hat{D}_{2}, \Phi_{2}$ is a positive definite design parameter matrix.

Then we can design the control torques $\boldsymbol{\tau}^{b}$ and parameter update law for $D_{2}$ as

$$
\begin{gathered}
\tau^{b}=-J\left[c_{2} e_{2}-J^{-1}(\Omega \times J \Omega)-J^{-1} G_{a}-\dot{\Omega}_{c}+W^{\mathrm{T}} \bar{e}_{1}+\beta_{2} \hat{D}_{2}\right] \\
\dot{\hat{D}}_{2}=\Phi_{2}^{-1} \beta_{2}^{\mathrm{T}} e_{2}
\end{gathered}
$$

where $c_{2}$ is a positive definite matrix to be designed.

Based on (21), (24) and (25), we have

$$
\begin{aligned}
\dot{\bar{e}_{1}} & =W \Omega+\beta_{1} D_{1}-\dot{\xi}_{\mathrm{c}}+c_{1} \varepsilon-W\left(\Omega_{c}-\Omega_{d}^{*}\right) \\
& =W \Omega_{d}^{*}+W\left(\Omega-\Omega_{c}\right)+\beta_{1} D_{1}-\dot{\xi}_{\mathrm{c}}+c_{1} \varepsilon \\
& =-c_{1} \bar{e}_{1}+\beta_{1} \tilde{D}_{1}+W e_{2}
\end{aligned}
$$

Using the expression (29) and substituting it into (18) yields

$$
\dot{e}_{2}=-c_{2} e_{2}+\beta_{2} \tilde{D}_{2}-W^{\mathrm{T}} \bar{e}_{1}
$$

Substituting (26), (30)-(32) into (28), we obtain

$$
\dot{\mathrm{V}}_{2}=\bar{e}_{1}^{\mathrm{T}} c_{1} \bar{e}_{1}-e_{2}^{\mathrm{T}} c_{2} e_{2}<0
$$

Thus, the attitude and angular rate tracking errors $\bar{e}$ and $e_{2}$ converge to zero exponentially.

\section{SimUlATION RESUlTS AND DISCUSSION}

In this section, in order to verify the validity and efficiency of the control law and update laws for the composite 
disturbances $D_{i},(i=1,2)$, simulations of a typical trajectory tracking task is performed on Matlab/Simulink.

The hexarotor UAV model parameters are taken as $m=1.7 \mathrm{Kg}, \quad g=9.81 \mathrm{~m} / \mathrm{s}^{2}, \quad l_{1}=281.5 \mathrm{~mm}, \quad l_{2}=287.6 \mathrm{~mm}$, $l_{3}=40.08 \mathrm{~mm}, \alpha=45^{\circ}, J=\operatorname{diag}\{0.0019,0.0019,0.031\} \mathrm{Kg} . \mathrm{m}^{2}$, $J_{r}=3.357 \times 10^{-5} \mathrm{Kg} \cdot \mathrm{m}^{2}, \quad b=2.98 \times 10^{-6} \mathrm{~N} \mathrm{~s}^{2} / \mathrm{rad}^{2}, d=1.14 \times 10^{-}$ ${ }^{7} \mathrm{Nm} \mathrm{s} / \mathrm{rad}^{2}$.

In the simulation, the initial positions and attitude angles are $\boldsymbol{p}_{0}=(0,0,0)^{\mathrm{T}}, \boldsymbol{\xi}_{0}=(0,0,0)^{\mathrm{T}}$, respectively, and so are linear and angular velocities, respectively. The composite disturbances inputs are chosen as $D_{1}(t)=[0.05,0.1,0.15]^{\mathrm{T}}$, $D_{2}(t)=[0.1 \cos (0.5 \mathrm{t}), 0.01 \sin (0.4 \mathrm{t})+0.1,0.05 \cos (0.3 \mathrm{t})+0.15]^{\mathrm{T}}$. And the disturbance matrices $b_{i},(i=1,2)$ are all unit matrix.

The desired horizontal rectangle trajectory is given by:

$$
\left\{\begin{aligned}
x_{c}= & 0.8(t-5) \mathrm{fsg}(t, 5,10)+4 \mathrm{fsg}(t, 10,15)+ \\
& 0.8(20-t) \mathrm{fsg}(t, 15,20) \\
y_{c}= & 0.6(t-10) \mathrm{fsg}(t, 10,15)+3 \mathrm{fsg}(t, 15,20)+ \\
& 0.6(25-t) \mathrm{fsg}(t, 20,25) \\
z_{c}= & 0.6 t \mathrm{fsg}(t, 0,5)+3 \mathrm{fsg}(t, 5,30) \\
\psi_{c}= & 0.2 \mathrm{rad}
\end{aligned}\right.
$$

where fsg is an interval function and expressed as $\operatorname{fsg}(x, a, b)=\frac{\operatorname{sign}(x-a)-\operatorname{sign}(x-b)}{2}$.

TABLE I. COMMAND FILTER PARAMETERS

\begin{tabular}{cccc}
\hline Parameter & $\omega_{n}$ & Mag.limit & Rate.limit \\
\hline$p$ & $2 \times 10^{-3}$ & $\pm 5 \mathrm{rad} / \mathrm{s}$ & $\pm 10 \mathrm{rad} / \mathrm{s}^{2}$ \\
$q$ & $2 \times 10^{-3}$ & $\pm 5 \mathrm{rad} / \mathrm{s}$ & $\pm 10 \mathrm{rad} / \mathrm{s}^{2}$ \\
$r$ & $2 \times 10^{-3}$ & $\pm 5 \mathrm{rad} / \mathrm{s}$ & $\pm 10 \mathrm{rad} / \mathrm{s}^{2}$
\end{tabular}

The position controller and attitude controller parameters in simulations are fixed at $K_{p}=0.98, K_{d}=0.265, K_{x}=1.0$, $K_{y}=0.97, c_{1}=\operatorname{diag}\{2,2,2\}, c_{2}=\operatorname{diag}\{3,3,3\}$. The update law parameters are chosen as $\Phi_{1}=\operatorname{diag}\{0.2,0.2,0.2\}, \Phi_{2}=\operatorname{diag}\{0.05,0.05,0.05\}$ and the command filter parameters are shown in Table 1.

The comparison of the simulation results is demonstrated in Fig.5 and Fig.7 where dashed-dot-dot lines represent the reference trajectory and the desired attitude angles, the solid lines correspond to the responses with update laws, and the dashed lines represent the responses without update laws. The detailed time responses for the trajectories of $x, y, z$ direction are presented in Fig. 6.

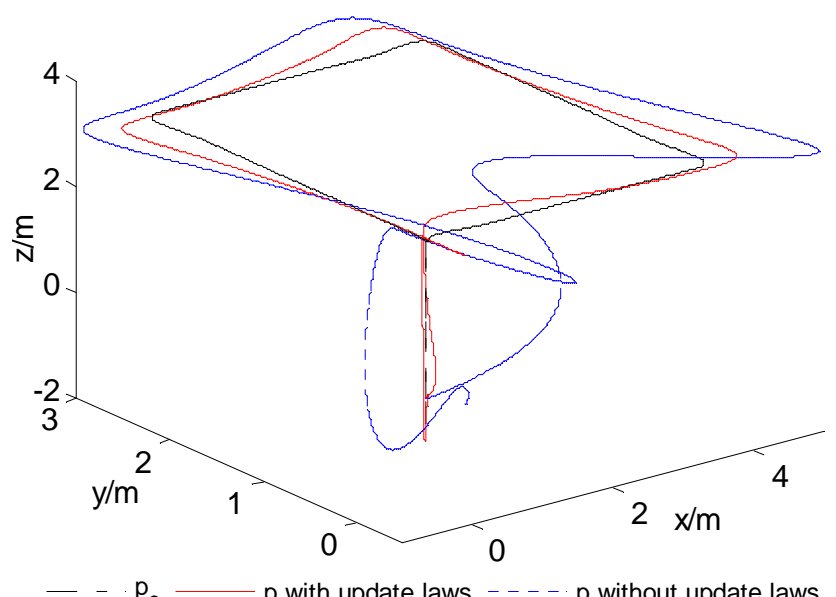

$-\overline{-} \cdot \overline{\text { Actual flight trajectory with update laws }}$
...... : Actual flight trajectory without update laws FIGURE V. THE 3D FLIGHT TRAJECTORY AND DESIRED TRAJECTORY
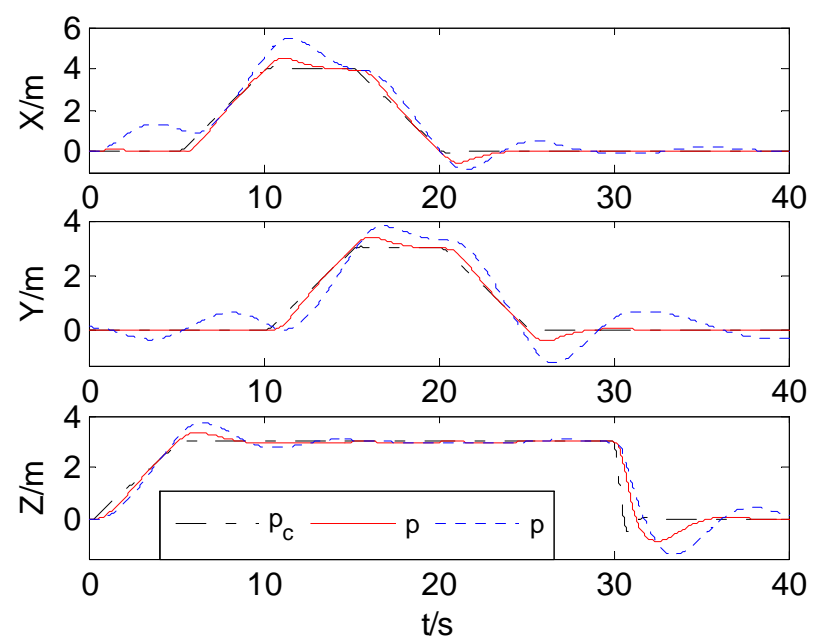

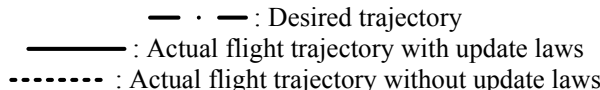

FIGURE VI. ACTUAL TRAJECTORY AND DESIRED TRAJECTORY OF $\mathrm{X}, \mathrm{Y}, \mathrm{Z}$ DIRECTION

From Fig. 5, the trajectory tracking without the update laws seems to work fine; however, the desired performance is not obtained. In Fig. 6, the trajectories of $x, y, z$ direction and the responses of the attitude angles exhibit significant deviations. Contrarily, the actual flight trajectory with the update laws closely follows the desired trajectory even under the composite disturbances. So, it is obviously that the control law performs better with the update laws under the composite disturbances inputs. At the same time, the simulation results verify that the proposed control strategy has the same efficiency to track different trajectory types. 

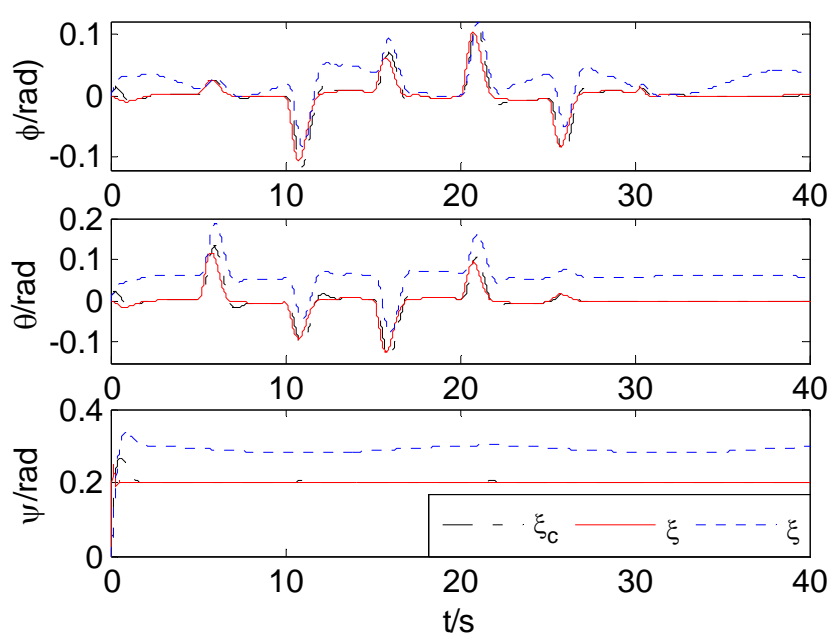

— - — : Desired attitude angles produced by Eq.(21) — : Responses of attitude angles with update laws -......- : Responses of attitude angles without update laws

FIGURE VII. RESPONSES OF THE ATTITUDE ANGLES FOR TRACKING THE DESIRED TRAJECTORY

\section{CONCLUSIONS}

An adaptive command filtered backstepping control strategy is applied for the trajectory tracking problem of an under-actuated and strong coupling nonlinear hexarotor model with verification by the experiments. The hexarotor rotational dynamics is developed to include the composite disturbances. Update laws are established for the composite disturbances which include aerodynamic moments, external disturbance and parameter uncertainties. Simulation results demonstrate that the proposed control strategy provides desirable trajectory tracking performance levels even under the composite disturbances inputs.

\section{ACKNOWLEDGMENT}

This work was supported by the Open Research Fund of Jiangsu Collaborative Innovation Center for Smart Distribution Network(XTCX201714), Nanjing Institute of Technology and High-level Scientific Research Foundation for the Introduction of Talent of Nanjing Institute of Technology (YKJ201412).

\section{REFERENCES}

[1] E. Altug, J. P. Ostrowski, C. J. Taylor. Control of helicopter using dual camera visual feedback. The International Journal of Robotics Research, 2005, 24(5): 329-341.

[2] H. Bouadi, M. Bouchoucha, M. Tadhine. Sliding mode control based on backstepping approach for an UAV type-quadrotor. International Journal of Applied Mathematics \& Computer Sciences, 2008, 4(1): 12-17.

[3] A. A. Mian, D. B. Wang, Modeling and backstepping based nonlinear control strategy for a 6 DOF quadrotor helicopter. Chinese Journal of Aeronautics, 2008, 21(3): 261-268. (in Chinese)

[4] Z. Zuo. Trajectory tracking control design with command-filtered compensation for a quadrotor. IET Control Theory and Applications, 2010, 4(11): 2343-2355.

[5] A. Das, K. Subbarao, F. Lewis. Dynamic inversion with zero-dynamic stabilization for quadrotor control. IET Control Theory and Application, 2009, 3(3): 303-314.
[6] D. Lee, H. J. Kim, S. Sastry. Feedback linearization vs. adaptive sliding mode control for a quadrotor helicopter. International Journal of Control, Automation and System, 2009, 7(3): 419-428.

[7] B. T. Whitehead, S. R. Bieniawski. Model reference adaptive control of a quadrotor UAV. AIAA Guidance, Navigation and Control Conference, Toronto, Ontario Canada, August 2-5, 2010.

[8] D. Swaroop, J. K. Hedrick, P. P. Yip, J. C. Gerdes. Dynamic surface control for a class of nonlinear systems, IEEE Transactions on Automatic Control, 2000, 45(10): 1893- 1899.

[9] D. Wang, J. Huang. Neural network-based adaptive dynamic surface control for a class of uncertain nonlinear systems in strict feedback form, IEEE Transactions on Neural Networks, 2005, 16(1): 195-202.

[10] J. A. Farrell, M. Polycarpou, M. Sharma, W. Dong. Command filtered backstepping, IEEE Transactions on Automatic Control, 2009, 54(6): 1391-1395.

[11] J. A. Farrell, M. Polycarpou, M. Sharma, Longitudinal flight path control using on-line function approximation, AIAA Journal of Guidance, Control and Dynamics, 2003, 26(6): 885-897.

[12] C. S. Yang, Z. Yang, D. Z. Xu, L. Ge. Trajectory tracking control for a novel six rotor aircraft. Systems Engineering and Electronics, 2012, 34(10): 2098-2105. (in Chinese) 